\title{
Ferroelectricity and isotope effects in hydrogen-bonded KDP crystals
}

\author{
S. Koval ${ }^{(1,2)}$, J. Kohanoff,(3), R. L. Migoni ${ }^{(2,1)}$, and E. Tosatti ${ }^{(1,4)}$ \\ 1) International Centre for Theoretical Physics, \\ Strada Costiera 11, I-34014 Trieste, Italy \\ 2) Instituto de Física Rosario, Universidad Nacional de Rosario, \\ 27 de Febrero 210 Bis, 2000 Rosario, Argentina. \\ 3) Atomistic Simulation Group, The Queen's University, Belfast BT7 1NN, Northern Ireland. \\ 4) International School for Advanced Studies (SISSA), \\ and Istituto Nazionale Fisica della Materia (INFM), Via Beirut 4, I-34014 Trieste, Italy
}

(October 24, 2018)

\begin{abstract}
Based on an accurate first principles description of the energetics in H-bonded KDP, we conduct a first study of nuclear quantum effects and of the changes brought about by deuteration. Cluster tunneling involving also heavy ions is allowed, the main effect of deuteration being a depletion of the proton probability density at the O-H-O bridge center, which in turn weakens its protonmediated covalent bonding. The ensuing lattice expansion couples selfconsistently with the proton off-centering, thus explaining both the giant isotope effect, and its close connection with geometrical effects.
\end{abstract}

Potassium dihydrogen phosphate $\left(\mathrm{KH}_{2} \mathrm{PO}_{4}\right.$, or KDP $)$ belongs to a family of ferroelectric (FE) crystals where molecular units are linked by hydrogen bonds, the ferroelectricity being connected to proton off-center ordering in the bonds. A characteristic feature of this family is the large increase in the Curie temperature $\mathrm{T}_{c}$ upon deuteration. In this particular case, it goes from $\simeq 122 \mathrm{~K}$ in $\mathrm{KDP}$ to $\simeq 229 \mathrm{~K}$ in the deuterated compound (DKDP). The origin of this huge isotope effect is still controversial, and has been mostly understood in terms of the quantum tunneling model proposed by Blinc, [1] later modified by inclusion of the coupling between proton motion and the $\mathrm{K}-\mathrm{PO}_{4}$ dynamics. 20 While direct experimental indications of tunneling have recently emerged [3], the connection between proton tunneling and isotope effect remains unclear. There is in fact strong evidence, [4.5] that isotope substitution acts rather through a geometrical modification of the hydrogen bonds, [6] with an expansion of the $\mathrm{O}-\mathrm{H}-\mathrm{O}$ distance. The proton off-centering, and thus the corresponding increase of lattice parameter upon deuteration, appear to be remarkably correlated to the increase of order parameter and of $\mathrm{T}_{c}$. These findings stimulated new theoretical work where some of these facts could be addressed without invoking tunneling, [7] however so far only at a rather phenomenological level.

In the first part of this letter we investigate, using electronic structure calculations within Density Functional Theory (DFT), the relationship between proton ordering, polarization, and geometry in KDP. In the second part, we introduce a study of energy and subsequently the quantization of the collective ion displacements in small KDP clusters, embedded in a host paraelectric lattice. These calculations demonstrate the difference between deuterated and protonated KDP, the more delocalized proton bridging the oxygens and pulling them together more effectively than the deuteron. This phenomenon, which is at the root of the geometric effect, is further illustrated by solving in the last part a selfconsistent nonlinear model.

For the DFT calculations we use two different approaches: one employing a basis set of confined pseudoatomic orbitals (SIESTA), [B] another a plane wave (PPW) representation. [9] For the first we choose a double-zeta basis set with polarization functions, and an orbital confinement energy $E_{c}=50 \mathrm{meV}$. In the second, we set the energy cutoff to 150 Ry. In both cases exchange-correlation terms are computed using a gradient-corrected (GGA) functional, 10 and normconserving pseudopotentials [1] are employed to represent the interaction between ionic cores and valence electrons. We also include the nonlinear core corrections for a proper description of the $\mathrm{K}$ ion. The Brillouin zone $\Gamma$-point alone provides a sufficient sampling in the large supercells used.

The paraelectric (PE) structure of KDP is bodycentered tetragonal with two $\mathrm{KH}_{2} \mathrm{PO}_{4}$ units per lattice

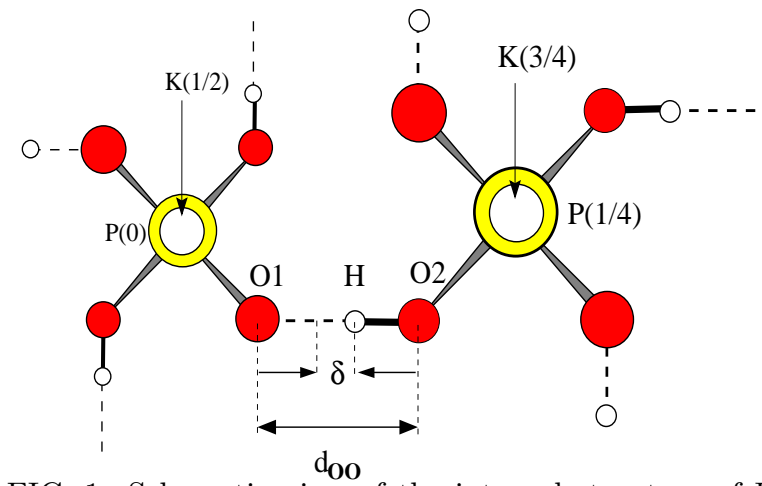

FIG. 1. Schematic view of the internal structure of KDP along the tetragonal axis. $\mathrm{P}$ and $\mathrm{K}$ coordinates relative to $c$ along this axis are indicated. Covalent and H-bonded hydrogens in the FE phase are attached to the corresponding oxygen through full and broken lines, respectively. 
site. We use the conventional bct cell doubled along the tetragonal $c$ axis (64 atoms) to describe homogeneous distortions, and a conventional $f c t$ cell doubled along the $c$-axis (128 atoms) for local distortions. The internal structure is depicted in Fig. 1. Above $\mathrm{T}_{c}$ the protons occupy with equal probability two equivalent off-center positions in the H-bond separated by a distance $\delta$, 12] while below $\mathrm{T}_{c}$ they order in such a way that each $\mathrm{PO}_{4}$ group has two covalently bonded and two H-bonded hydrogen atoms.

We first analyse the relationship between proton ordering and polarization. We start from the average experimental structure of the PE phase of KDP at $\mathrm{T}_{c}^{K D P}+5 \mathrm{~K}$, 112 with the hydrogens centered in the $\mathrm{O}-\mathrm{H}-\mathrm{O}$ bonds. By fully relaxing the atomic positions in the bct cell, the $\mathrm{H}$ atoms move collectively off-center towards the $\mathrm{O} 2$ oxygens, and away from O1, as indicated in Fig. 1. Analysis of Mulliken populations and charge density differential maps [13] indicates that the off-centering of the $\mathrm{H}$ atoms induces a covalent charge displacement from $\mathrm{O} 2$ towards the $\mathrm{O} 2-\mathrm{H}$ bonds, whereas the $\mathrm{O} 1-\mathrm{H}$ bonds weakens into a hydrogen bond. In addition, there is a charge flow from the O2-P to the O1-P bonds, accompanied by an increase of the $\mathrm{P}-\mathrm{O} 2$ and a decrease of the $\mathrm{P}-\mathrm{O} 1$ distances. $\mathrm{P}$ atoms are thus driven off-center in the $\mathrm{PO}_{4}$ tetrahedra, which polarize further. Unbalanced electrostatic forces induce a displacement of the $\mathrm{K}^{+}$ions along the $c$-axis, towards the charge-excess (O1) side of $\mathrm{PO}_{4}$ units. A detailed description of the classical FE distortion in KDP can be found in Ref. [13]. These results are also in agreement with Ref. [14]. In order to identify the driving mechanism of the $\mathrm{FE}$ instability, we investigate the $a b$ initio potential energy surface (PES) as a function of the proton off-centering parameter $\delta=d_{O O}-2 d_{O H}$, and of the K-P relative displacement along $c, \gamma=c-2 d_{K P}$, which provides a measure of the polarization. 115 By fully relaxing the oxygen positions for each $\operatorname{chosen}(\delta, \gamma)$ pair, we obtain a two-dimensional double-well PES with a saddle point at $\delta=\gamma=0$, whose contours are reported in the inset to Fig. 2. According to this PES, the crystal is stable against polarization $(\gamma \neq 0)$ unless the protons are ordered off-center $(\delta \neq 0)$. In Fig. 2 we show cuts of the PES at different values of $\gamma$, indicating that, even for vanishing $\gamma$, the energy minimum corresponds to a finite $\delta$, i.e. protons are always collectively unstable at the $\mathrm{H}$ bond centers. Therefore, we confirm that the source of the FE instability is indeed the $\mathrm{H}$ off-centering.

In order to address next the quantum effects, in particular the isotope substitution, we need to introduce quantum fluctuations in the above classical picture. Barring for the time being a full brute force quantum mechanical calculation for all the ionic degrees of freedom of the infinite system, we take a different approach to the problem. Important quantum effects are identified as those involving correlated $\mathrm{H}$ motions (as shown in Fig. 1) with relaxation of $\mathrm{K}$ and $\mathrm{P}$ ions, most favorable for exhibiting

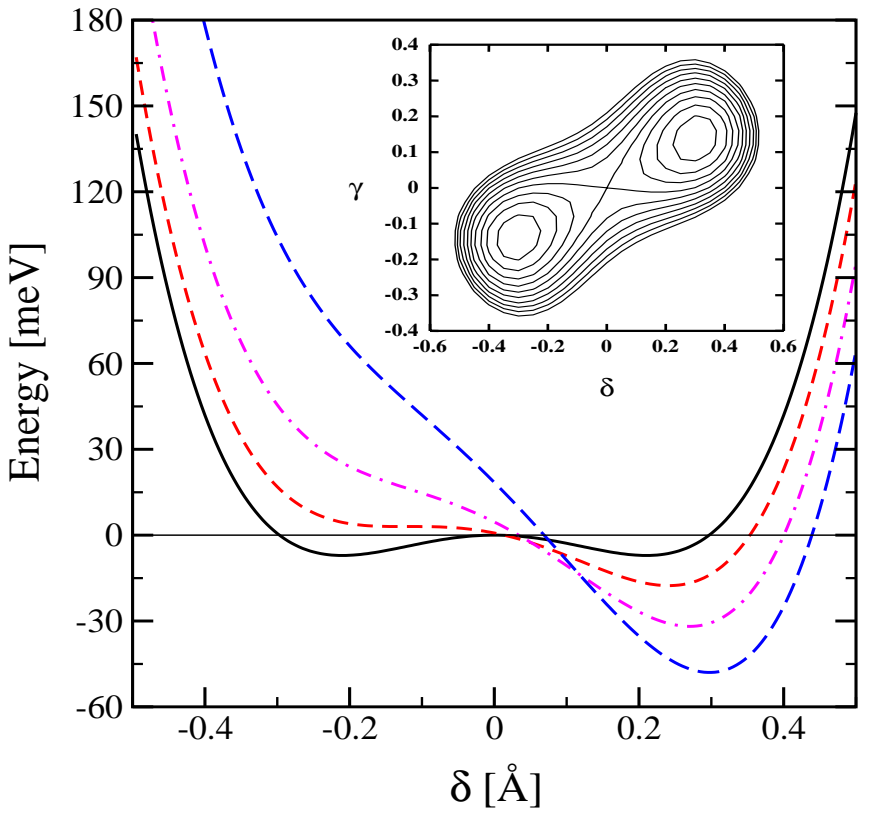

FIG. 2. Energy profiles as a function of $\delta$, for values of $\gamma=0$ (solid line), 0.02 (short-dashed), 0.05 (dot-dashed) and 0.1 (long-dashed) $\AA$. The minima are always at $\delta \neq 0$, and for $\gamma \geq 0.02 \AA$ the curves exhibit a single minimum. The inset shows equispaced energy contours (step $=13.6 \mathrm{meV} /$ $\mathrm{KH}_{2} \mathrm{PO}_{4}$ unit $)$. The minima at $(\delta, \gamma) \simeq \pm(0.3,0.15) \AA$ lie $\simeq 50$ meV below the saddle point at $(0,0)$.

FE instabilities. We shall consider this correlated pattern as a single collective coordinate $\delta_{c}$. Quantization of this coordinate will moreover be carried out within a finitesize cluster. Even though quantum fluctuations span in principle all length scales, those that occur at short range should be sufficiently revealing at least away from critical points.

In this spirit, we consider a series of small KDP clusters of increasing size, embedded inside an undistorted host PE matrix. For these clusters, we first determine, classically, the total energy variation as a function of $\delta_{c} / 2$. The clusters comprise $\mathrm{N}$ hydrogens, in the following order : (a) $\mathrm{N}=1$; (b) $\mathrm{N}=4$, fully connecting a $\mathrm{PO}_{4}$ group to the host; (c) $\mathrm{N}=7$, connecting two $\mathrm{PO}_{4}$ groups; (d) $\mathrm{N}=10$, connecting three $\mathrm{PO}_{4}$ groups. In order to ascertain the effect of the volume increase seen upon deuteration, we repeat the calculations by expanding the host structural parameters to the corresponding experimental values of DKDP at $\mathrm{T}_{c}^{D K D P}+5 \mathrm{~K}$. 12] The results show (Fig. 3, solid curves) that there is an instability only when the cluster size is sufficiently large, thus providing a measure of the FE correlation length. The instability is much stronger (note the larger energy scale), and the correlation length accordingly shorter, with the expanded structural parameters of DKDP. This is in close agreement with the experimental trend, showing that the FE order grows with volume. Finally, the involvement of $\mathrm{P}$ and $\mathrm{K}$ motions is important. The dashed curves in 
Fig. 3 represent the energy of a distortion where only $\mathrm{H}$ atoms are allowed to move, all other atoms being kept fixed. The much higher energies indicate that, not surprisingly, this kind of oversimplified distortion is far from real, leaving, e.g., the KDP lattice fully stable.

The next step in order to study the quantum effects due to proton or deuteron zero point motion, is to quantize the clusters with respect to the local collective coordinate $\delta_{c} / 2$. The corresponding kinetic energy involves the mass of each ion proportionally to the square of its displacement, and will change upon deuteration. We find that the deuterium (hydrogen) effective mass for this correlated motion is about $\mu_{D}=3.0\left(\mu_{H}=2.3\right)$ proton masses in DKDP (KDP) clusters. Solving Schrödinger's equation for the single variable $\delta_{c} / 2$ with mass $\mu_{H, D}$ and effective potentials of Fig. 3 is trivial. The ground state (GS) energy levels which lie below the energy barrier at $\delta_{c} / 2=0$ are indicated by dotted lines. A negative energy signals the occurrence of tunneling between + and $-\delta_{c} / 2$, and its onset provides a rough indication of the correlation volume. The results show that it comprises more than $\mathrm{N}=10$ hydrogens in KDP, but no more than $\mathrm{N}=4$ deuteriums in DKDP. In both systems, we eventually expect the collective tunnel splitting to converge to zero as $N \rightarrow \infty$, signaling long-range ferroelectricity; the tunnel splitting should conversely remain nonzero in the quantum paraelectric state, attainable at high pressure. (5, 17

Remarkably, deuteration at fixed geometry does not affect the fact that the tunnel splittings in large clusters [16] are exceedingly smaller than $\mathrm{k}_{B} \mathrm{~T}_{c}$, which renders $\mathrm{T}_{c}$ practically insensitive. In addition, the effective mass change is reduced by the involvement of the heavy nuclei. This agrees with high-pressure experiments, [5. 17 . where under fixed structural conditions the isotope effect appears to be only very slight.

The fact that energy barriers in DKDP are much larger than those in KDP implies that quantum effects are significantly reduced in the expanded DKDP lattice. After this observation, the reason for the geometric effect becomes clear: in all KDP clusters considered here the wavefunction $(\mathrm{WF})$ for the collective coordinate $\delta_{c}$ is much more delocalized, with a larger amplitude near the $\mathrm{H}$-centred position $\delta_{c}=0$ between $\mathrm{O} 1$ and $\mathrm{O} 2$, than in DKDP. In KDP in other words, zero-point motion pushes protons towards the center, much more effectively so than deuterons in DKDP. An increased probability for the proton to bridge midway between the two oxygens is not irrelevant to the crystal cohesion, and we identify precisely that as the element which compresses the cell from a larger classical value to the smaller value found for KDP. To estimate an upper limit to that effect, we compare the lattice parameter and the $\mathrm{O}-\mathrm{H}-\mathrm{O}$ bridge length $d_{O O}$ of two classical electronic calculations: one with the hydrogens forced to sit in the bridge center, the other with $\mathrm{H}$ fully off-center in the classical FE state. The result is

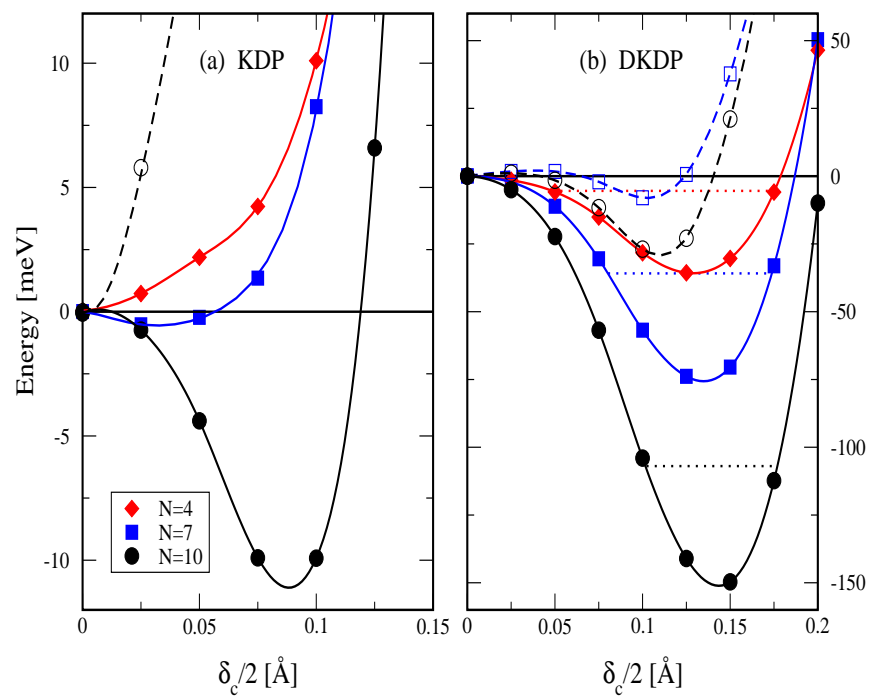

FIG. 3. Ab initio energy profiles for classical distortions of clusters embedded in undistorted PE structures of (a) KDP and (b) DKDP. Reported are clusters of: $\mathrm{N}=4$ (diamonds), $\mathrm{N}=7$ (squares), and $\mathrm{N}=10$ (circles). Full symbols and solid lines: $\mathrm{P}$ and $\mathrm{K}$ positions inside the cluster are allowed to relax as $\mathrm{H}$ are off-centered; empty symbols and dashed lines: only $\mathrm{H}$ atoms are moved. Dotted lines: GS energies (only negative ones, signaling tunneling).

$4.42 \AA$ when $\mathrm{H}$ is centered. At the equilibrium volume, we estimate that proton centering creates an equivalent pressure of approximately $20 \mathrm{kbar}$. Thus, a centered hydrogen acts as a very strong attraction center that pulls the two bridge oxygens together, the whole lattice volume shrinking by $2.3 \%$ as a result. In the true hightemperature $\mathrm{PE}$ phase the hydrogens are of course not exactly centered, but appear on both sides of the H-bond, thus reducing the magnitude of the effect.

Deuteration of KDP to DKDP at fixed geometry results in a depleted probability for the deuterium to reach the bridge center, as shown in Fig 4(a) where we plot the proton and deuteron WF in the DKDP potential of the $\mathrm{N}=7$ cluster of Fig. 3(b). This depletion releases a fraction of the O-H-O bond grip, causing a small lattice expansion which has the effect of increasing the potential wells depth, making the deuteron even more localized, and so on in a self-consistent manner. The overall self-consistent effect is eventually much larger than the mere fixed-geometry replacement of the proton with the deuteron mass. In fact, for the same cluster size in KDP, instead of a double-peak, the WF exhibits a broad single peak centered in $\delta_{c} / 2=0$ (see Fig. 4(a)). This mechanism is now capable of explaining, at least qualitatively, the increase in the order parameter and $\mathrm{T}_{c}$, which are related to the mass only indirectly, through the geometric modification of the length and energy scales.

We constructed a simple model that demonstrates the nonlinear behaviour arising from isotope substitution in KDP by adding to the effective hydrogen potential in the 

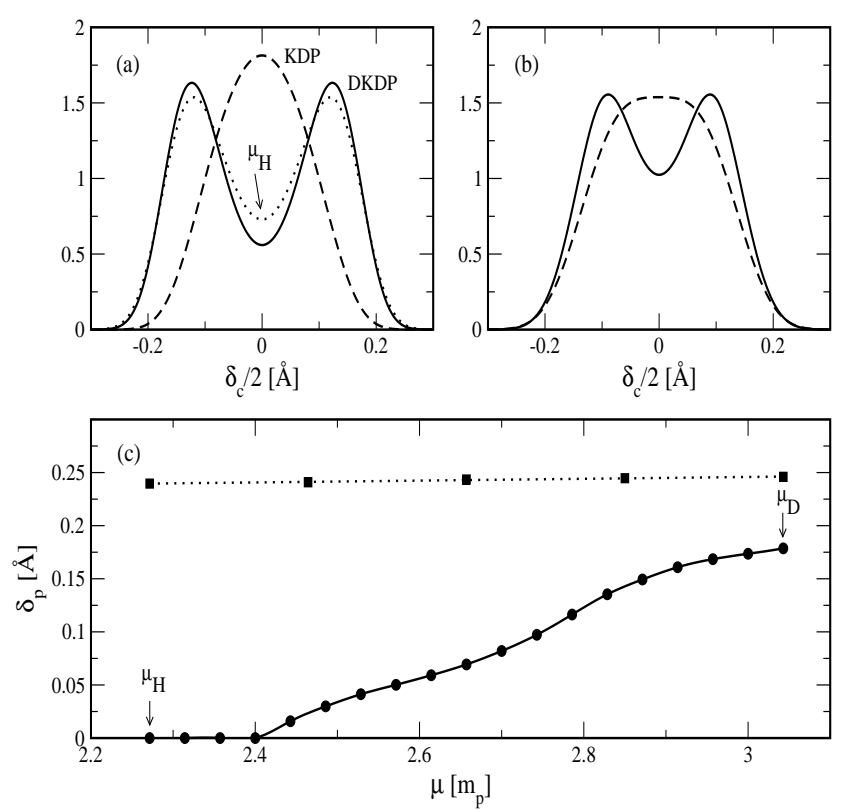

FIG. 4. WF in the $\mathrm{N}=7$ cluster PES for (a) ab initio and (b) self-consistent model calculations. Solid (dashed) lines are for DKDP (KDP). Dotted line is for $\mathrm{H}$ in the DKDP PES. (c) WF peak separation $\delta_{p}$ as a function of the effective mass $\mu$ (given in units of the proton mass) for the self-consistent model (circles) and for fixed DKDP potential (squares). Lines are guides to the eye.

cluster Schrödinger equation a quadratic WF-dependent term of the form $V_{\text {eff }}(x)=V_{0}(x)-k|\Psi(x)|^{2}$, where $x=$ $\delta_{c} / 2$ and $V_{0}(x)$ is a quartic double-well similar to those of Fig. 3. The nonlinear $|\Psi(x)|^{2}$ term signifies the positive feedback discussed above, since a decreasing mass will increase $|\Psi(x)|^{2}$ at the center, which will in turn lower the barrier at that point, further increasing $|\Psi(x)|^{2}$, and so on. By choosing appropriately $k$ and the "bare" potential $V_{0}(x)$, we find that the corresponding $a b$ initio profiles for KDP and DKDP can be qualitatively reproduced (Fig. 4 (b)). The self-consistent solution evolves from a doublepeak to a single-peak situation when changing the mass from "pure DKDP" to "pure KDP", as shown by the circles in Fig. 4 (c). Such a large mass dependence, in striking contrast with the very weak dependence obtained at fixed DKDP potential and geometry (squares), can now explain the large and controversial isotope effect in the ferroelectricity of KDP.

Summarizing, we confirm that the $\mathrm{H}$ off-center ordering is the source of FE instability. First principles calculations in a model PE phase show that distortions involving only $\mathrm{H}$ atoms do not display significant instabilities. Instead, heavy cluster tunneling involving also $\mathrm{K}$ and $\mathrm{P}$ ions, explains the "double occupancy" in the PE phase, in agreement with the observation of P-ion double-site distribution. 18] Although tunneling is of course the crucial ingredient in the isotope effect, its mere change at fixed lattice geometry accounts only for a very small fraction of the full isotope effect. The main effect of replacing deuterons with protons appears to be an enhancement of the quantum delocalization of the proton in the bond center region. That gives rise to a strong proton-mediated covalency in the O-H-O bridge, which shrinks the lattice, which further delocalizes the proton, and so on in a nonlinear loop. In the end, the huge isotope effect on $\mathrm{T}_{c}$ is dominated by the geometrical effect. This effect, however, is triggered by tunneling, thus reconciling these two aspects which were largely debated in the past.

We thank R.J. Nelmes, M.I. McMahon, R. Resta, A. Bussmann-Holder, G. Colizzi, G. Reiter, M.G. Stachiotti and D. Marx for helpful discussions. R.M. and S.K. thank support from CONICET, Argentina, and from ICTP, Trieste. E.T.'s work was also supported by MIUR COFIN01, and by INFM/G.

[1] R. Blinc, J. Phys. Chem. Solids 13, 204 (1960).

[2] K. Kobayashi, J. Phys. Soc. Jpn. 24, 497 (1968); E. Matsushita and T. Matsubara, Prog. Theor. Phys. 67, 1 (1982); T. Matsubara and E. Matsushita, Prog. Theor. Phys. 71, 209 (1984); M. Kojyo and Y. Onodera, J. Phys. Soc. Jpn. 57, 4391 (1988); A. Bussmann-Holder and K.H. Michel, Phys. Rev. Lett. 80, 2173 (1998).

[3] Compton scattering experiments appear to provide evidence for proton tunneling (G. Reiter, private commun.).

[4] M. Ichikawa, K. Motida and N. Yamada, Phys. Rev. B 36, 874 (1987); Z. Tun et al., J. Phys. C: Solid State Phys. 21, 245 (1988); R.J. Nelmes, ibid. 21, L881 (1988).

[5] M.I. McMahon et al., Nature (London) 348, 317 (1990).

[6] J.M. Robertson and A.R. Ubbelohde, Proc. R. Soc. London A 170, 222 (1939).

[7] H. Sugimoto and S. Ikeda, J. Phys.: Condens. Matter. 8, 603 (1996)

[8] P. Ordejón, E. Artacho and J.M. Soler, Phys. Rev. B 53, R10441 (1996); D. Sánchez-Portal et al., Int. J. Quantum Chem. 65, 453 (1997).

[9] C. Cavazzoni and G.L. Chiarotti, Computer Phys. Commun. 123, 56 (1999).

[10] J.P. Perdew, K. Burke and M. Ernzerhof, Phys. Rev. Lett. 77, 3865 (1996).

[11] N. Troullier and J.L. Martins, Phys. Rev. B 43, 1993 (1991).

[12] R.J. Nelmes, Z. Tun and W.F. Kuhs, Ferroelectrics 71, 125 (1987).

[13] S. Koval et al., Comput. Mater. Science 22, 87 (2001).

[14] Q. Zhang et al., Phys. Rev. B 65, 024108 (2002).

[15] J. Kohanoff, S. Koval and R. Migoni (unpublished).

[16] For the nearly second order FE transition in KDP (see G.A. Samara, Ferroelectrics 5, 25 (1973)), large clusters are the relevant ones.

[17] R.J. Nelmes et al., Ferroelectrics 124, 355 (1991).

[18] M.I. McMahon et al., Europhys. Lett. 13, 143 (1990). 